\title{
The Usefulness of a Wearable Device in Daily Physical Activity Monitoring for the Hospitalized Patients Undergo- ing Lumbar Surgery
}

\author{
Dong Hwan Kim, Kyoung Hyup Nam, ${ }^{1}$ Byung Kwan Choi, ${ }^{1}$ In Ho Han, ${ }^{1}$ Tae Jin Jeon, ${ }^{2}$ Se Young Park ${ }^{3}$ \\ Department of Neurosurgery \& Medical Research Institue, Pusan National University Hospital, Pusan National University School of Medicine, Busan, Korea \\ SOOSANG ST Co., Inc., ${ }^{2}$ Busan, Korea \\ Department of Computer Science and Engineering, ${ }^{3}$ Pusan National University, Busan, Korea
}

Objective : Functional outcomes have traditionally been evaluated and compared using subjective surveys, such as visual analog scores (VAS), the Oswestry disability index (ODI), and Short Form-36 (SF-36), to assess symptoms and quality of life. However, these surveys are limited by their subjective natures and inherent bias caused by differences in patient perceptions of symptoms. The Fitbit Charge ${ }^{\circledast}$ (Fitbit Inc., San Francisco, CA, USA) provides accurate and objective measures of physical activity. The use of this device in patients after laminectomy would provide objective physical measures that define ambulatory function, activity level, and degree of recovery. Therefore, the present study was conducted to identify relationships between the number of steps taken by patients per day and VAS pain scores, prognoses, and postoperative functional outcomes.

Methods : We prospectively investigated 22 consecutive patients that underwent laminectomy for spinal stenosis or a herniated lumbar disc between June 2015 and April 2016 by the same surgeon. When patients were admitted for surgery and first visited after surgery, preoperative and postoperative functional scores were recorded using VAS scores, ODI scores, and SF-36. The VAS scores and physical activities were recorded daily from postoperative day (POD) 1 to POD 7. The relationship between daily VAS scores and daily physical activities were investigated by simple correlation analysis and the relationship between mean number of steps taken and ODI scores after surgery was subjected to simple regression analysis. In addition, Wilcoxon's signed-rank test was used to investigate the significance of pre-to-postoperative differences in VAS, ODI, and SF-36 scores.

Results : Pre-to-postoperative VAS $(p<0.001)$, ODI $(p<0.001)$, SF-36 mental composite scores $(p=0.009)$, and SF-36 physical composite scores $(p<0.001)$ scores were found to be significantly different. Numbers of steps taken from POD 1 to POD 7 were negatively correlated with daily VAS scores $(r=-0.981, p<0.001)$. In addition, the mean number of steps from POD 3 to POD 7 and the decrease in ODI conducted one month after surgery were statistically significant $(p=0.029)$.

Conclusion : Wearable devices are not only being used increasingly by consumers as lifestyle devices, but are also progressively being used in the medical area. This is the first study to demonstrate the usefulness of a wearable device for checking patient physical activity and predicting pain and prognosis after laminectomy. Based on our experience, the wearable device used to provide measures of physical activity in the present study has the potential to provide objective information on pain severity and prognosis.

Key Words : Wearable electronic device · Visual analog scale · Laminectomy · Exercise.

- Received : June 15, 2018 •Revised : August 6, 2018 •Accepted : September 19, 2018

- Address for reprints : In Ho Han

Department of Neurosurgery, Pusan National University Hospital, 179 Gudeok-ro, Seo-gu, Busan 49241, Korea

Tel : +82-51-240-7257, Fax : +82-51-244-0282, E-mail : farlateral@hanmail.net, ORCID : https://orcid.org/0000-0001-7193-6533

This is an Open Access article distributed under the terms of the Creative Commons Attribution Non-Commercial License (http://creativecommons.org/licenses/by-nc/4.0) which permits unrestricted non-commercial use, distribution, and reproduction in any medium, provided the original work is properly cited. 


\section{INTRODUCTION}

The internet of things (IoT) is a network of physical devices, vehicles, buildings, and other items embedded with electronics, software, sensors, actuators, and network connectivity that enable these devices to collect and exchange data ${ }^{9)}$. Wearable devices such as activity trackers, which are a good example of the IoT, have been widely used in the healthcare field $^{2,6,14,17)}$, and wearable devices for the real-time monitoring of biological information have received considerable attention in the medical field ${ }^{8,10,12,18)}$.

Monitoring of biological patient information is particularly important in hospital settings. Information regarding the daily physical activities of patents, such as ambulation, is important in the contexts of patient care and nursing ${ }^{11,17)}$. However, no objective scale has been proposed for monitoring of daily physical activity. We considered a suitable wearable device could provide medical staff with objective information on daily activities that would aid assessments of patient conditions and outcomes, particularly after spinal surgery.

Therefore, the present study was conducted to investigate the usefulness of wearable devices for the assessment of patients' daily physical activities in a hospital environment and for exploring relationships between physical activity, postoperative pain and short-term surgical outcomes.

\section{MATERIALS AND METHODS}

\section{Ethics approval}

This study was approved by the Institutional Review Board of Pusan National University Hospital (IRB No. 1606-004042). All patients provided written informed consent.

\section{Participants}

This study was conducted using a consecutive, prospective design. Patients were enrolled between June 2015 and April 2016 by the surgeon that performed the surgical procedure. The inclusion criteria were the receipt of partial laminectomy at one level because of spinal stenosis or a lumbar herniated disc causing low back pain, radiating leg pain, and gait disturbance. The exclusion criteria included history of lumbar or leg surgery or the presence of any comorbid condition, such as osteoporotic compression fracture, tumor, infection, motor weakness, or chronic kidney disease capable of affecting physical activity after surgery. Patients not prepared to comply with study requirements, those with poor memory or a mental health issue, and those who would not provide informed consent were excluded.

\section{Wearable device}

We used the Fitbit Charge ${ }^{\circledR}$ (Fitbit Inc., San Francisco, CA, USA), which is one of the most common wireless physical activity monitors available to consumers, to obtain physical activity performance data ${ }^{1}$. This unit is a small, lightweight, and simple device, which was worn by subjects on an elastic band around the waist at hip level on one side (left or right) throughout the study. The unit has a battery life of 7-10 days and was therefore able to monitor physical activity for a week on a single charge. Patients were advised to remove the device only when there was a risk of it being in contact with water (e.g., while washing).

The unit is equipped with a three-dimensional accelerometer, altimeter, and vibration motor that converts acceleration to step counts using an inbuilt algorithm and can estimate numbers of steps taken, flights of stairs climbed, distance walked, and calories expended. Collected data can be stored online on a password protected website and accessed by user login. The device synchronizes wirelessly when placed within $6 \mathrm{~m}$ of an active USB dongle ${ }^{19)}$. The accuracy of the Fitbit device has been demonstrated in various reports ${ }^{3,7,19)}$.

In the present study, the unit was used to record average physical activity from postoperative day (POD) 1 to POD 7. All data (number of steps taken per day, distance traveled per day, and calories burned per day) were obtained from units after POD 7. Particular emphasis was placed on numbers of steps taken per day and distance traveled per day.

\section{Traditional self-reported survey}

Patient clinical symptoms and outcomes were measured using a patient-based scoring system. When patients were admitted for surgery or visited the hospital 1 month after surgery, preoperative and postoperative functional scores were recorded in the form of visual analog scores (VAS) (pain), the Oswestry disability index (ODI), Short Form-36 (SF-36) mental composite scores (MCS), and physical composite scores (PCS). The VAS scores were recorded daily from POD 1 to POD 7. 


\section{Statistics}

Statistical analysis was performed using SPSS (version 18; IBM, Armonk, NY, USA). Demographic variables including age and gender were summarized using descriptive statistics (as means \pm standard deviations or as percentages). The relationship between postoperative daily VAS pain scores and postoperative physical activity (with focus on the number of steps taken daily from POD 1 to POD 7) was assessed by simple correlation analysis to determine whether a significant correlation existed between daily VAS scores and numbers of steps taken daily. The results of Pearson's correlation analysis are presented as $r$ and $p$ values, where $r$ values signify strengths of correlations ( $\mathrm{r}$ values close to 1 indicate a strong positive correlation, while values closer to -1 indicate a strong negative correlation).

Relationships between the mean numbers of steps from POD 3 to POD 7 and changes in clinical outcome measures (VAS, ODI, and SF-36 MCS and PCS) were analyzed by simple regression analysis. In addition, Wilcoxon's signed-rank test was used to identify significant differences between preoperative and postoperative VAS, ODI, or SF-36 scores. A $p$ value $<0.05$ was considered to indicate significance.

\section{RESULTS}

Twenty-seven patients were included in the present study, and 22 patients completed accelerometer physical activity motoring and postoperative self-reported surveys (VAS, ODI, and SF-36 MCS and PCS) during clinical follow-up. The physical activities of two of the 27 patients were not measured using a Fitbit Charge unit because one patient did not wear the unit and the device did not work for the other. In addition, three patients did not complete postoperative self-reported surveys because they did not visit the hospital during follow-up. Accordingly, these five patients were excluded. The mean age of the 22 patients that comprised the study cohort was 59.64士 13.42 years and 11 were male (50\%). In addition, seven patients had spinal stenosis, 15 had a lumbar herniated disc, and all 22 underwent one level partial laminectomy by the same surgeon. Symptoms were only back pain $(n=4)$, only radiating pain $(n=6)$, and back pain with radiating pain $(n=12)$ (Table 1$)$.

The results of pre- and post-operative self-reported surveys are summarized in Table 2. For all 22 study subjects, and mean VAS score at latest follow up visits (1 month postoperatively) decreased significantly from $7.4 \pm 2.17$ to $1.4 \pm 1.94$ $(p<0.001)$, while the mean ODI score decreased significantly from $58.6 \pm 20.03$ to $22.5 \pm 16.66(p<0.001)$. Additionally, the mean SF-36 PCS increased significantly from $35.4 \pm 14.59$ to $60.6 \pm 17.57$ at follow-up $(p<0.001)$, while the SF-36 MCS increased significantly from $51.5 \pm 16.68$ to $65.9 \pm 15.34(p<0.009)$. For all 22 patients, preoperative to postoperative changes in self-reported surveys were significant.

The mean number of daily steps taken by the 22 study subjects gradually increased from $773 \pm 1199.49$ on POD 1 to 5412 \pm 2712.44 on POD 7 (Table 3 ), the mean distance walked increased from $558 \pm 864 \mathrm{~m}$ to $3909 \pm 1941 \mathrm{~m}$, and the VAS scores gradually decreased from $6.59 \pm 2.11$ to $2.32 \pm 1.43$. Simple correlation analysis was used to evaluate the relationship between

Table 1. Demographic data of participants

\begin{tabular}{lc}
\hline & Value \\
\hline Mean age (years) & $59.64 \pm 13.42$ \\
Gender & 11 \\
Male & 11 \\
Female & \\
Pathology & 7 \\
Spinal stenosis & 15 \\
Herniatied disc & $7.36 \pm 2.17$ \\
Preoperative pain (VAS) & \\
Symptom & 4 \\
Back pain & 6 \\
Radiating pain & 12 \\
Back pain with radiating pain &
\end{tabular}

Values are presented as mean \pm standard deviation or number. VAS: visual analogue scales

Table 2. Traditional self-reported survey of preoperative and postoperative result

\begin{tabular}{lcr}
\hline & $\begin{array}{c}\text { Preoperative } \\
\text { result }\end{array}$ & Postoperative result \\
\hline VAS & $7.4 \pm 2.17$ & $1.4 \pm 1.94(p<0.001)$ \\
ODI & $58.6 \pm 20.03$ & $22.5 \pm 16.66(p<0.001)$ \\
SF-36 & & \\
Mental composite score & $51.5 \pm 16.68$ & $65.9 \pm 15.34(p<0.009)$ \\
Physical composite score & $35.4 \pm 14.59$ & $60.6 \pm 17.57(p<0.001)$ \\
\hline
\end{tabular}

Values are presented as mean \pm standard deviation. VAS : visual analogue scale, ODI : oswestry disability index, SF-36 : short form health survey-36 
daily VAS scores and physical activity over POD 1 to 7 (mean VAS scores and mean number of steps taken daily are provided in Table 3). The analysis showed a significant, negative, linear correlation between the two ( $\mathrm{r}=-0.981, p<0.001)$ (Fig. 1).

The relationship between physical activity during hospitalization and short-term surgical outcome (VAS, ODI, and SF36) at 1 month postoperatively was also investigated. The mean number of steps from POD 3 to POD 7 and the decrease in ODI conducted 1 month after surgery were statistically significant $(p=0.029)$.

Table 3. Average value of VAS, number of steps, and distance from POD 1 to POD 7

\begin{tabular}{lccc|}
\hline & VAS & Number of steps & Distance $(\mathrm{m})$ \\
\hline POD 1 & $6.59 \pm 2.11$ & $772.95 \pm 1199.49$ & $558 \pm 864$ \\
POD 2 & $5.45 \pm 1.99$ & $1524.27 \pm 2232.13$ & $1101 \pm 1603$ \\
\hline POD 3 & $4.68 \pm 1.55$ & $2040.73 \pm 1498.26$ & $1469 \pm 1073$ \\
POD 4 & $4.00 \pm 1.15$ & $3081.82 \pm 1982.68$ & $2232 \pm 1428$ \\
\hline POD 5 & $3.64 \pm 1.68$ & $3790.68 \pm 2006.05$ & $2742 \pm 1470$ \\
POD 6 & $3.05 \pm 1.70$ & $4032.64 \pm 2334.21$ & $2916 \pm 1695$ \\
\hline POD 7 & $2.32 \pm 1.43$ & $5411.77 \pm 2712.44$ & $3909 \pm 1941$ \\
\hline
\end{tabular}

Values are presented as mean \pm standard deviation. VAS : visual analogue scale, POD : postoperative day

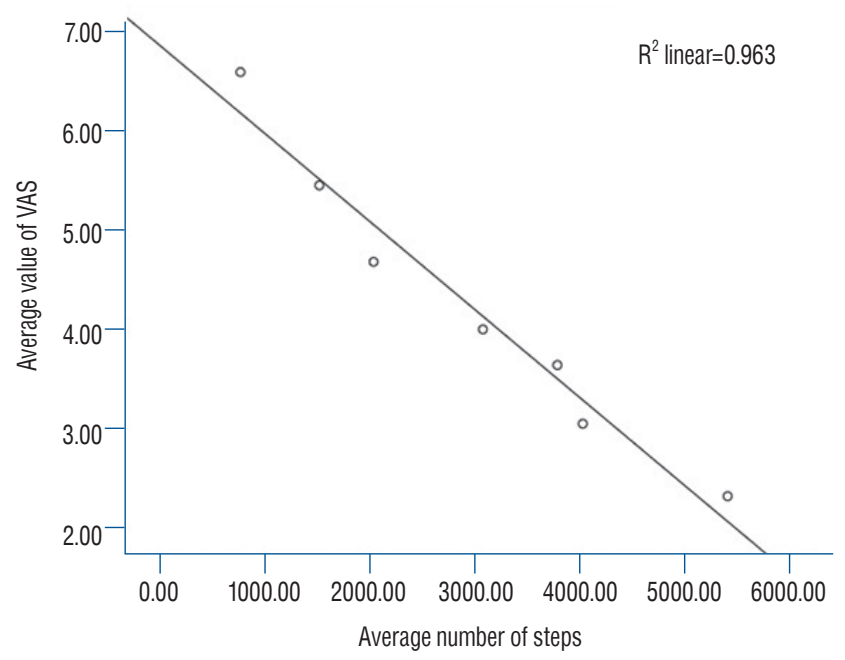

Fig. 1. Negative linear correlation of mean value of VAS and number of steps from POD 1 to POD 7. VAS : visual analog scores, POD : postoperative day.

\section{DISCUSSION}

The results of this study demonstrated that increases in daily physical activity are significantly related to reductions in daily VAS pain scores after surgery, indicating that postoperative ambulation is closely related to postoperative pain.

This finding means that measures of daily physical activity acquired from wearable devices provide reliable information. If daily physical activities are included in medical records, doctors and nurses could use this information to improve the care and monitoring of patients. For example, medical staff could exactly monitor the extent of daily ambulation or bed rest and educate patients accordingly.

As daily activity is closely related to postoperative VAS scores, surgeons could also predict immediate surgical outcomes or early complications such as postoperative infection or hematoma by analyzing changing daily activity patterns. In addition, daily physical activity during hospitalization was found to be significantly related to short-term surgical outcomes, especially ODI at 1 month postoperatively, which suggests daily physical activity monitoring during hospitalization may enable the prediction of short-term and immediate outcomes. The functional outcomes of spine patients are closely related to walking activity ${ }^{4,11,17)}$; thus, wearable devices offer the potential to obtain objective functional information after discharge and to compare pre- and postoperative physical activities. Several trials have been conducted to measure objective physical activity using a wireless accelerometer in spine patients. For example, Mobbs et al. ${ }^{13)}$ measured functional outcomes in patients after lumbar surgery using a quantitative measure of physical activity acquired using an accelerometer. They found that the mean number of steps and distance travelled increased at 3 months postoperatively, but no significant correlation was found between improvements in subjective clinical outcome scores and changes in physical activity measurements during follow-up ${ }^{13)}$. Pryce et al..$^{17)}$ reported subjective measures of pain and disability had limited abilities to account for real-time activities measured using an accelerometer in patients with lumbar spinal stenosis. The limitation of wireless accelerometers is that measures of physical activity are limited to walking activity. However, walking activity is the main component of physical activity in the ODI and SF-36 test; therefore, monitoring of walking activities is worthwhile for assessing functional outcomes. In addition, technological 
developments of wearable devices could further expand measures of physical activity and replace subjective self-report scales $^{5}$.

Sensor, machine learning, and wireless communication technology developments are continuously improving and increasing the multi-functionalities of wearable devices, and it is likely that wearable devices will be used to monitor hospitalized patients for various purposes in the near future ${ }^{15,16}$. For example, these devices can immediately detect an accident, such as a fall, and alert doctors or nurses. They could also be used to detect the unexpected escape of dementia or delirious patients, and to prevent bed sores of the over-immobilization of quadriplegic and paraplegic patients by educating them on the important of position changing.

There are some limitations in this study. Sample size is small and follow-up period is short. However, Hospitalization of long period over 1 week is difficult for patients who underwent one level laminectomy. We studied the hospitalized patients to minimize bias as much as possible. Compliance to wearable devices could be low in outpatients. So we studied the patients during hospitalized to increase compliance and accuracy. Based on the present result, we plan to study the application of wearable device for outpatient during further period over 1 week.

\section{CONCLUSION}

This is the first study to demonstrate wearable devices can be used to monitor the objective physical activities of patients in the hospital after spine surgery. The wearable device used was found to provide an objective measure of serial physical activity and to provide information that would aid the monitoring and care of hospitalized patients by medical staff. In the near future, we believe multifunctional wearable medical devices could be used to monitor patients in real-time.

\section{CONFLICTS OF INTEREST}

No potential conflict of interest relevant to this article was reported.

\section{INFORMED CONSENT}

Informed consent was obtained from all individual participants included in this study.

\section{AUTHOR CONTRIBUTIONS}

\author{
Conceptualization : IHH, BKC \\ Data curation : DHK, KHN, TJJ, SYP \\ Formal analysis : DHK, KHN \\ Funding acquisition : BKC \\ Methodology : DHK, KHN, IHH, TJJ, SYP \\ Project administration : $\mathrm{IHH}$ \\ Visualization : DHK \\ Writing - original draft : DHK \\ Writing - review \& editing : IHH
}

\section{References}

1. Adam Noah J, Spierer DK, Gu J, Bronner S : Comparison of steps and energy expenditure assessment in adults of Fitbit tracker and ultra to the actical and indirect calorimetry. J Med Eng Technol 37 : 456-462, 2013

2. Berlin JE, Storti KL, Brach JS : Using activity monitors to measure physical activity in free-living conditions. Phys Ther 86 : 1137-1145, 2006

3. Cadmus-Bertram LA, Marcus BH, Patterson RE, Parker BA, Morey BL : Randomized trial of a Fitbit-based physical activity intervention for women. Am J Prev Med 49 : 414-418, 2015

4. Chapman JR, Norvell DC, Hermsmeyer JT, Bransford RJ, DeVine J, McGirt MJ, et al. : Evaluating common outcomes for measuring treatment success for chronic low back pain. Spine (Phila Pa 1976) 36(21 Suppl) : S54-S68, 2011

5. Chen KY, Bassett DR Jr : The technology of accelerometry-based activity monitors: current and future. Med Sci Sports Exerc 37(11 Suppl) : S490-S500, 2005

6. Culhane KM, O'Connor M, Lyons D, Lyons GM : Accelerometers in rehabilitation medicine for older adults. Age Ageing 34 : 556-560, 2005

7. Diaz KM, Krupka DJ, Chang MJ, Peacock J, Ma Y, Goldsmith J, et al. : Fitbit $\AA$ : an accurate and reliable device for wireless physical activity tracking. Int J Cardiol 185 : 138-140, 2015

8. Dimitrov DV : Medical internet of things and big data in healthcare. Healthc Inform Res 22 : 156-163, 2016

9. Gershenfeld N, Krikorian R, Cohen D : The internet of things. Sci Am $291: 76-81,2004$

10. Hao A, Wang $L$ : Medical device integration model based on the internet 
of things. Open Biomed Eng J 9 : 256-261, 2015

11. Iversen MD, Katz JN : Examination findings and self-reported walking capacity in patients with lumbar spinal stenosis. Phys Ther 81 : 12961306, 2001

12. Metcalf $D$, Milliard ST, Gomez M, Schwartz $M$ : Wearables and the internet of things for health: wearable, interconnected devices promise more efficient and comprehensive health care. IEEE Pulse 7 : 35-39, 2016

13. Mobbs RJ, Phan K, Maharaj M, Rao PJ : Physical activity measured with accelerometer and self-rated disability in lumbar spine surgery: a prospective study. Global Spine J 6 : 459-464, 2016

14. Noorkõiv $\mathrm{M}$, Rodgers $\mathrm{H}$, Price $\mathrm{Cl}$ : Accelerometer measurement of upper extremity movement after stroke: a systematic review of clinical studies. J Neuroeng Rehabil 11 : 144, 2014

15. Pantelopoulos A, Bourbakis NG : A survey on wearable sensor-based systems for health monitoring and prognosis. IEEE Trans Syst Man
Cybern $40: 1-12,2010$

16. Park $S$, Jayaraman $S$ : Enhancing the quality of life through wearable technology. IEEE Trans Inf Technol Biomed 22 : 41-48, 2003

17. Pryce R, Johnson M, Goytan M, Passmore S, Berrington N, Kriellaars D : Relationship between ambulatory performance and self-rated disability in patients with lumbar spinal stenosis. Spine (Phila Pa 1976) 37 : 1316-1323, 2012

18. Scheer JK, Bakhsheshian J, Keefe MK, Lafage V, Bess S, Protopsaltis TS, et al. : Initial experience with real-time continuous physical activity monitoring in patients undergoing spine surgery. Clin Spine Surg 30 : E1434-E1443, 2017

19. Takacs J, Pollock CL, Guenther JR, Bahar M, Napier C, Hunt MA : Validation of the Fitbit one activity monitor device during treadmill walking. J Sci Med Sport 17 : 496-500, 2014 